\title{
An Anatomicosurgical Study of the Temporal Branch of the Facial Nerve
}

\author{
- Mario Ammirati, M.D., Aldo Spallone, M.D., \\ Jianya Ma, M.D., Mel Cheatham, M.D., \\ Donald Becker, M.D.
}

Division of Neurosurgery, University of California, Los Angeles, California (MA, JM, MC, DB); Division of Neurosurgery, University of Rome

"Tor Vergata," Rome, Italy (AS)

THE SURGICAL ANATOMY of the temporal branch of the facial nerve was studied bilaterally in 10 embalmed cadaveric heads. Particular attention was paid to the relationships between the temporal branch, the galeal-fascial layers, and the fat pads of the temporal-zygomatic region. The temporal branch of the facial nerve pierces the parotidomasseteric fascia below the zygomatic arch. This branch travels first in the subcutaneous tissue and then, above the zygomatic arch, in the subgaleal space. The temporal branch divides into an anterior, a middle (frontal), and a posterior ramus soon after it pierces the parotid fascia. The course of the terminal twigs of the temporal branch of the facial nerve in the subgaleal space is extremely variable, with their location being at times posterior to the anterior one-fourth of the temporalis muscle. Occasionally, a twig for the frontalis muscle may run in between the two layers of the superficial temporal fascia. Because of these findings (anteroposterior variability of temporal branch twigs and recurrent intrafascial twig), Yasargil's interfascial dissection may at times fail. A combined frontotemporal scalp/superficial temporal fascia dissection is anatomically suited to preserve the temporal branch of the facial nerve. (Neurosurgery 33:1038-1044, 1993)

Key words: Facial nerve, Frontozygomatic region, Surgical anatomy, Temporal branch

$\mathbf{R}$ emoval of the zygomatic arch is becoming more and more common in approaching the vascular and neoplastic lesions in the cavernous sinus/petroclival area or in the interpeduncular fossa $(3,4,11,13,27)$. The risk of injuring the temporal branch of the facial nerve (frontotemporal branch of the facial nerve [29]), already known to be present in the simple pterional route, is increased in the transzygomatic approach because of the extensive soft tissue dissection necessary to completely expose the zygomatic arch. Injury of the temporal branch of the facial nerve may result in a cosmetically bothersome appearance as a result of paralysis of the frontalis, orbicularis oculi, and corrugator supercilii muscles. Preservation of the temporal branch of the facial nerve requires a clear understanding of its course and of its relationships with the multiple galeal-fascial layers of the temporal region. Yasargil (28) described the surgical anatomy relevant to the preservation of the temporal branch of the facial nerve during a pterional craniotomy. His recommendations have, however, generated results that are not always reproducible, probably as a result of the complex fascial anatomy of the temporal region (5, 9). The purpose of the present study is to define clearly the course of the temporal branch of the facial nerve and to study its relationships with the fascial planes of the pterional/zygomatic region to provide a clear anatomical base for its preservation during neurosurgical exposures.

\section{MATERIALS AND. METHODS}

Dissections were performed bilaterally in 10 embalmed cadaveric heads in which the cranial vascular system had been injected with silicone-colored material.

The subcutaneous tissue, galea, superficial temporal fascia, deep temporal fascia, temporalis muscle, parotidomasseteric fascia, and parotid gland were carefully dissected, and the facial nerve was exposed from the parotid region to the point where the uppermost ramus of its temporal branch enters the frontalis muscle. To evaluate objectively the individual variability of the temporal branch of the facial nerve, a line starting at the lateral canthus and ending at the tragus was arbitrarily chosen and two points were measured in reference to this line: 1) the bifurcation of the temporal branch of the facial nerve into an anterior and middle (frontal) ramus (28) (Fig. 1); and 2) the point where the 


\section{Temporal Branch of the Facial Nerve}

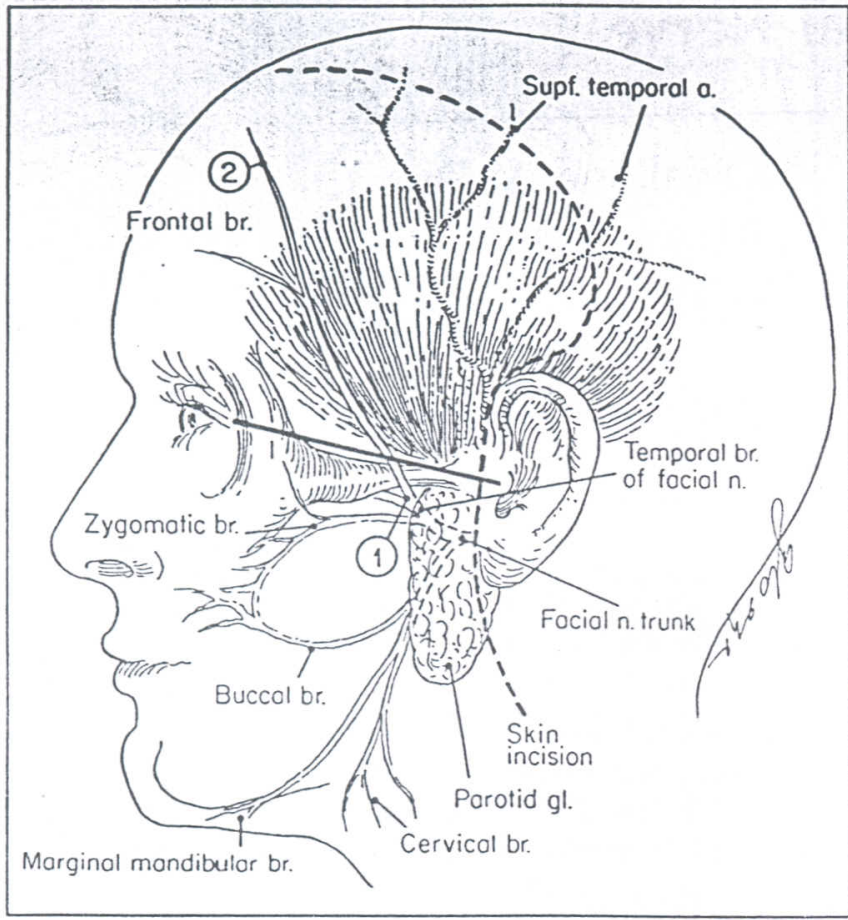

FIGURE 1. The skin flap, the reference line, and the peripheral course of the facial nerve are shown. The temporal branch of the facial nerve bifurcates below the zygomatic arch and below the reference line. 1, bifurcation of the temporal branch of the facial nerve into an anterior and middle (frontal) ramus; 2 , uppermost twig of the middle (frontal) ramus.

uppermost twig of the frontal ramus enters the frontalis muscle (Fig. 1).

\section{SURGICAL PROSECTION}

A question-mark skin incision starts in the frontal hair line close to the midline, extends backward in the posterior frontal region, and then curves downward to end below and in front of the ear (Fig. 1). The galea is dissected from the superficial temporal fascia in the line of the skin incision down to the zygomatic arch where the galea becomes continuous with the subcutaneous tissue. The parotidomasseteric fascia is then identified below the zygomatic arch. The facial nerve is exposed as it exits the stylomastoid foramen and then followed inside the parotid gland; its bifurcation into the cervicofacial and temporofacial branches is identified. Further, the division of the temporofacial into the zygomatic and temporal branch is exposed (Fig. 1). The bifurcation of the temporal branch of the facial nerve into an anterior and middle ramus (Fig. 1) and the uppermost twig of the middle (frontal) ramus are identified and their position noted with respect to the reference line. The

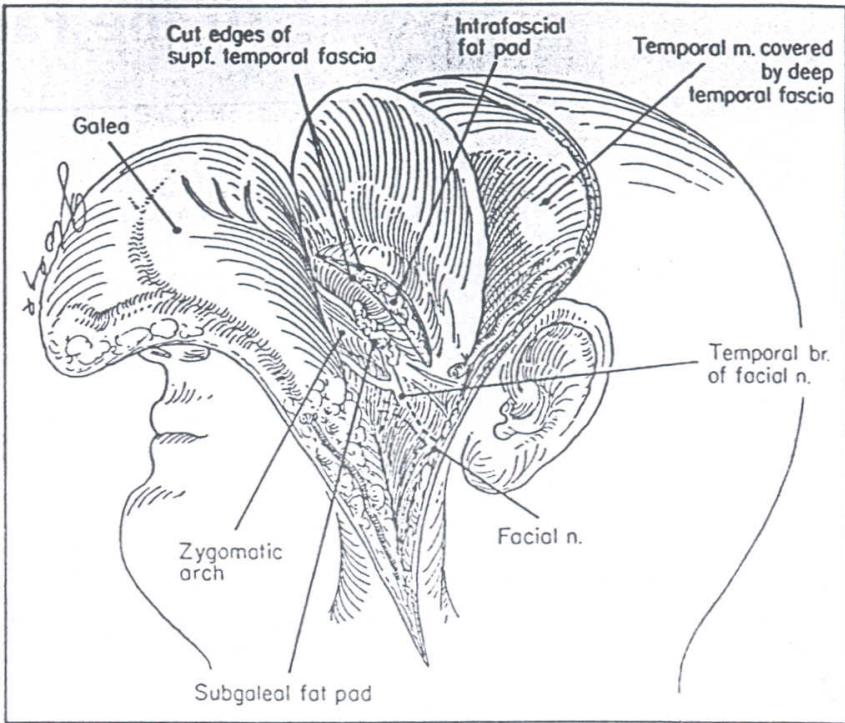

FIGURE 2. The intrafascial (between the two layers of the superficial temporal fascia) and the subgaleal fat pads are shown in this illustration. Above the zygomatic arch, the temporal branch of the facial nerve is located in the subgaleal space, intermixed with the subgaleal fat. It then pierces the galea to enter its target muscles.

point where the temporal branch of the facial nerve pierces the parotidomasseteric fascia to become subcutaneous is also noted. In exposing the whole course of the temporal nerve, a scalp flap is developed in the subgaleal space (Fig. 2). At this stage, the temporalis muscle covered by the superficial temporal fascia is fully exposed from the superior temporal line to its attachments on the lateral wall of the orbit and on the zygomatic arch. The superficial temporal fascia is incised at the upper border of the temporalis muscle and separated from the muscle. The insertions of the fascia on the frontozygomatic bone (frontal process of the zygomatic bone and zygomatic process of the frontal bone) and on the zygomatic arch are released. The duplication of this fascia over the anterior onefourth of the muscle is incised, and the fat pad concealed between these two layers is exposed (Fig. 3). The temporalis muscle covered by the thin deep temporal fascia is then separated from the frontozygomatic bone. The zygomatic arch may then be transected and the temporalis muscle retracted inferiorly, revealing the temporal fossa covered by its periosteum.

\section{RESULTS}

\section{Galeal-fascial layers}

The galea is present as a separate dissectable layer down to the zygomatic arch, where it blends with the subcutaneous tissue. However, the galea thins out significantly as the zygomatic arch is approached. The distal superficial temporal artery 


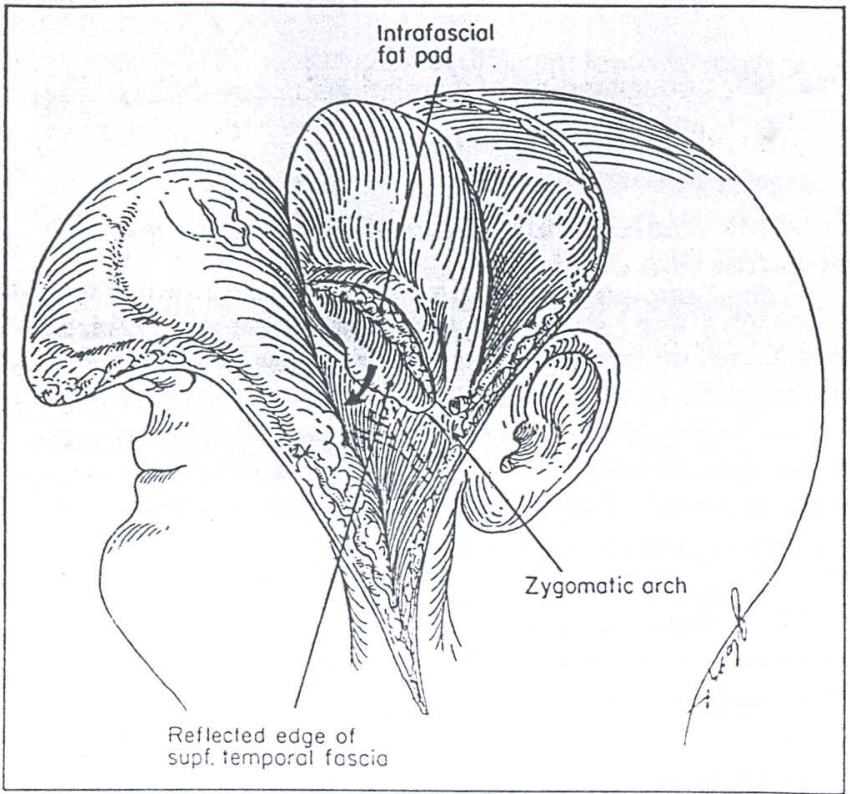

FIGURE 3. This illustration highlights the fat between the two layers of the superficial temporal fascia (intrafascial fat pad).

stem and its branches remain always in a subcutaneous plane, and the temporal branch of the facial nerve is always anterior to the superficial temporal artery trunk. In dissecting the scalp flap, either the posterior or the anterior division of the superficial temporal artery needs to be transected.

The superficial temporal fascia lies subgaleally and covers the temporalis muscle. It is a thick fascia, and it is always easily identifiable as a distinct layer. Superiorly and posteriorly, it attaches to the superior temporal line, being continuous with the periosteum (Fig. 4). Anteriorly, it attaches to the frontozygomatic bone, and inferiorly, it attaches to the zygomatic arch. Below the arch, it is continuous with the parotidomasseteric fascia. In the anterior one-fourth of this fascia, two layers can be recognized in between which there is a fat pad, a small artery and vein, and occasionally, a nerve twig (Fig. 3). The temporal branch of the facial nerve lies superficially to the superficial temporal fascia (Figs. 2, 3, 5). The two layers of this fascia attach to the anterior portion of the zygomatic arch and to the frontozygomatic bone in a parallel fashion, with the deep layer located behind the superficial one (Fig. 5 ). The deep temporal fascia is nothing else but a thin loose connective tissue sheath that envelopes the temporalis muscle (fascia propria). Beneath the temporalis muscle there is the periosteum of the temporal fossa. This periosteal layer is continuous superiorly, above the superior temporal line, with the frontoparietal periosteum (Fig. 4).

\section{Fat pads}

There are three fat pads over the anterior one-fourth of the temporalis muscle. There is a fat pad located in the subgaleal space that is continuous with the subcutaneous fat below the zygomatic arch (Fig. 2). The second fat pad, the temporal adi-

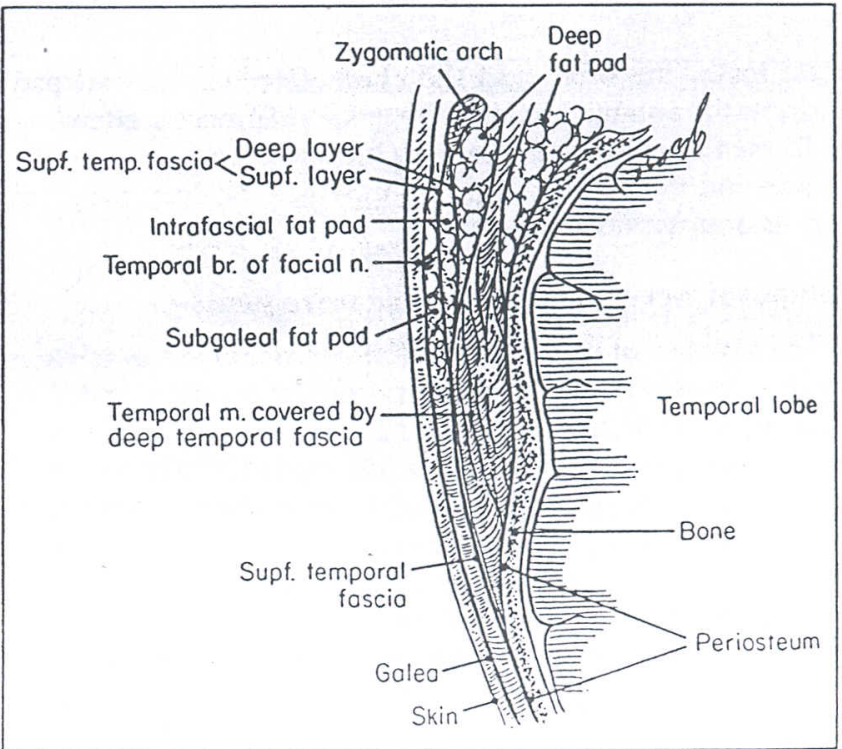

FIGURE 4. Diagrammatic representation of the different anatomical planes of the temporal fossa at the midzygomatic arch level, as seen in a coronal section. Note the position of the three fat pads, the galea that thins out toward the zygomatic arch, and the subgaleal location of the temporal branch of the facial nerve.

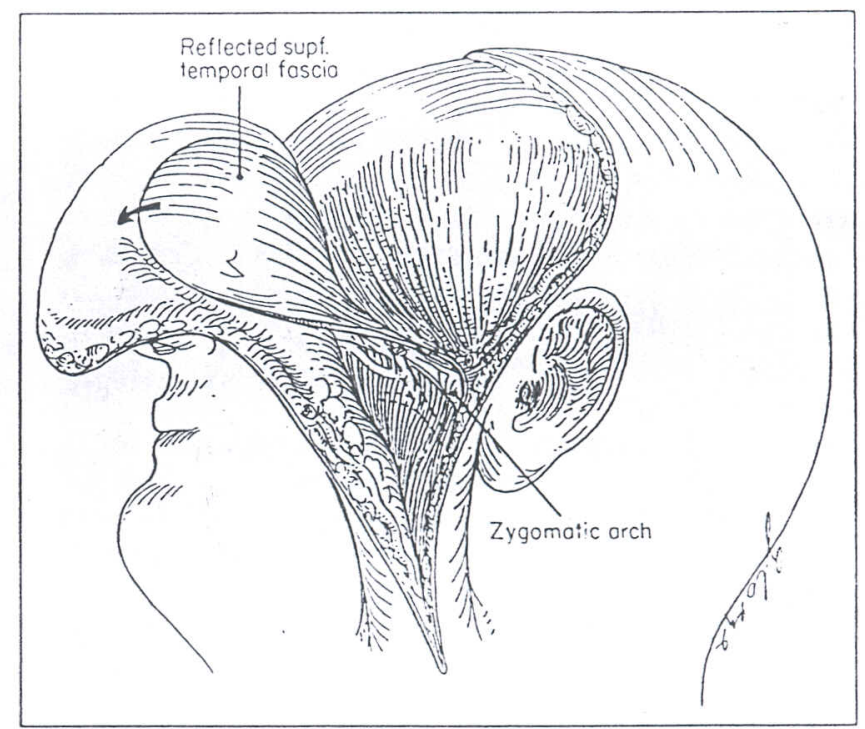

FIGURE 5. The superficial temporal fascia has been dissected free from the temporalis muscle. The temporal branch of the facial nerve is superficial to the superficial temporal fascia.

pose body of the German authors (19), is intrafascial, located in the duplication of the superficial temporal fascia (Figs. 2 and 3 ). The third fat pad is located beneath the superficial temporal fascia, between this fascia and the temporalis muscle. This fat pad surrounds the anterior-inferior portion of the temporalis muscle and is continuous with the fat tissue of the infratem- 
poral fossa, the orbit, and the check (Fig. 4). This fat pad encircles the anterior border of the temporalis muscle extending on its medial aspect and forming a fat-filled space between the muscle and the temporal squama in which the deep temporal vessels and nerves are located.

\section{Temporal nerve course and measurements}

The division of the temporofacial nerve into the zygomatic and temporal branch takes place inside the parotid gland (Fig. 1). The temporal branch of the facial nerve pierces the parotidomasseteric fascia always below the zygomatic arch and then immediately divides into its terminal rami-anterior, middle, and posterior (29). The anterior ramus innervates the corrugator supercilii and orbicularis oculi muscles, and the middle one (frontal ramus) innervates the frontalis muscle. The posterior ramus, for the variously developed anterior and superior auricular and tragus muscles, does not have any practical importance in man.

The point where the temporal branch of the facial nerve gives off the anterior and middle rami is located $1.1 \mathrm{~cm}$ (mean value) below the reference line (range, $0.5-1.9 \mathrm{~cm}$ ) and $2.4 \mathrm{~cm}$ (mean) anterior to the tragus (range, $1.5-3.5 \mathrm{~cm}$ ). The point where the uppermost twig of the frontal ramus pierces the galea to enter the frontalis muscle is located $2.4 \mathrm{~cm}$ (mean value) above the reference line (range, $1.0-3.7 \mathrm{~cm}$ ) and $1.5 \mathrm{~cm}$ (mean value) posterior to the lateral canthus (range, $0.5-3.6 \mathrm{~cm}$ ).

Relationships between the fat pads and the temporal nerve

The temporal branch of the facial nerve is located in the same plane as the superficial fat pad, both being in the subgaleal space. The temporal branch is located superficial to the intrafascial and to the deep fat pad (Figs. 2 and 3). However, a few times, we observed a sizable twig of the middle division of the temporal branch of the facial nerve (frontal ramus) going into the intrafascial space and then entering the frontalis muscle.

\section{DISCUSSION}

Relevance of the course of the temporal branch of the facial nerve to neurosurgical exposures

The temporal branch of the facial nerve is involved in the soft tissue dissection plane of all the approaches that involve retraction of the anterior portion of the temporalis muscle to expose the lateral cranial base of the anterior cranial fossa and of the middle fossa. Among others, the pterional approach and its numerous modifications $(2,16,21)$ as well as the various transzygomatic approaches $(3,4,11,13)$, all must deal with the temporal branch of the facial nerve.

Injury of the temporal branch of the facial nerve results in a cosmetically important paralysis of the frontalis, orbicularis oculi, and corrugator supercilii muscles. With the exception of the work done by Yasargil et al. (29), little or no attention has been paid in the neurosurgical literature to the course of the temporal branch of the facial nerve, while at the same time, the importance of its preservation has been recognized $(10,24)$. Preservation of the temporal branch of the facial nerve depends on a clear understanding of its relationships with the complex soft tissue anatomy of the temporal-zygomatic arch region.

\section{Previous studies dealing with the course of the temporal branch of the facial nerve}

Several studies in the ear, nose, and throat and plastic surgery literature have addressed the course of the temporal branch of the facial nerve and the strategies to preserve it (6-8, $12,15,17,20,22,23,25)$. Among others, Furnas (12) recommends staying above the temporal root of the zygoma, $1 \mathrm{~cm}$ posterosuperior to the anterior hairline at the zygomatic arch, and $2.0 \mathrm{~cm}$ posterosuperior to the lateral edge of the eyebrow. Pitanguy and Ramos (22) used a line connecting a point $0.5 \mathrm{~cm}$ below the tragus with a point $1.5 \mathrm{~cm}$ above the lateral extremity of the eyebrow to plot the course of the temporal branch of the facial nerve. Correia and Zani (8) located the temporal branch of the facial nerve in an area marked by two divergent lines extending from the the earlobe to the lateral end of the eyebrow and to the highest forehead crease, respectively. Finally, Bernstein and Nelson (7) in an accurate anatomical study of the course of the temporal branch of the facial nerve concluded that "the temporal branch of the facial nerve lies within an area bounded by a line from the earlobe to the lateral edge of the eyebrow inferiorly and a second line from the tragus to the lateral coronal suture just above and behind the highest forehead crease." Yasargil (29) described three rami of the temporal branch of the facial nerve, with the middle (for the frontalis muscle) and anterior (for the orbicularis oculi and corrugator supercilii muscles) rami running in the subcutaneous tissue over the zygomatic arch, $1 \mathrm{~cm}$ anterior to the superficial temporal artery. The posterior ramus is for the anterior and superior auricular and tragus muscles.

\section{Results of the present study}

Our finding of the presence of the galea as a separate dissectable layer that thins out as the zygomatic arch is approached is in agreement with previous reports $(14,26,28)$. Other authors refer to this superficial connective tissue layer of the temporal region as the temporoparietal fascia or superficial temporal fascia and describe it as being continuous superiorly, above the temporal region, with the galea, and inferiorly, below the zygomatic arch, with the superficial musculoaponeurotic system of the face (25).

The present study has shown a significant variability in the course of the temporal branch of the facial nerve. This variability is demonstrated by the wide range of each of the points selected with respect to the reference line. This finding is in accord with previous reports $(1,6,7,20)$. We have also found that the temporal branch of the facial nerve, when the parotidomasseteric fascia is pierced (always below the zygomatic arch), is at first located in the subcutaneous tissue because the galea does not extend below the zygomatic arch. However, above the arch, the temporal branch of the facial nerve is located deep to the galea, in the subgaleal space between the galea superficially and the superficial temporal fascia deeply. From there on, the terminal twigs of the temporal branch of the 
facial nerve penetrate the galea at different levels to reach their target muscles that are all located superficial to the galea.

Three fat pads were constantly individuated over the anterior portion of the temporalis muscle. The superficial one is located subgaleally and, therefore, in the same plane of the temporal branch of the facial nerve (Fig. 4). The intrafascial fat pad is located in the duplication of the superficial temporal fascia (Fig. 4), and the deep fat pad is located deep to the superficial temporal fascia, between this fascia and the temporalis muscle (Fig. 4).

The intrafascial and the deep fat pad are located in a different, deeper plane than the temporal branch of the facial nerve and therefore do not have any relationship with the temporal branch, with the significant exception of an occasional twig of the temporal branch that runs intrafascially to distally penetrate the frontalis muscle later on

Available techniques (and their pitfalls) to preserve the temporal branch of the facial nerve

The subgaleal dissection of a temporal scalp flap is associated with a high incidence of postoperative palsy of the temporal branch of the facial nerve. This is not surprising considering that the temporal branch runs in the subgaleal space.

Reflecting the scalp and temporalis muscle together as a single layer (myocutaneous flap) virtually eliminates the incidence of injury to the temporal branch of the facial nerve, at the expense, however, of compromised basal exposure resulting from the bulky temporalis muscle (29).

Yasargil $(28,29)$ proposed a subgaleal dissection up to the anterior one-fourth of the temporalis muscle where the dissection has to be deepened between the two layers of the superficial temporal fascia. The fat in between the two layers of the superficial temporal fascia marks the point where the dissection has to be deepened. This approach, although better than the subgaleal dissection, may still injure the temporal branch of the facial nerve in the subgaleal space because of the great variability along an anteroposterior line of the course of the terminal twigs of the temporal branch of the facial nerve. Furthermore, at times, as demonstrated by our study, there is a twig for the frontalis muscle that runs intrafascially just where the dissection, according to Yasargil's technique, has to take place. In addition confusion may arise between the subgaleal and the intrafascial fat pad. Obviously if one mistakes the subgaleal fat pad for the intrafascial one, injury to the temporal branch of the facial nerve is very likely. Moreover, it is relatively easy, when dissecting intrafascially, to "slip" from the intrafascial into the subgaleal plane (Fig. 6).

Recently Labbe et al. (18) described a surgical approach to the fronto-orbital and pterional region that detaches the temporalis muscle from the bone, using a subperiosteal, subtemporal cleavage plane. Although this approach is anatomically suited to preserve the temporal branch of the facial nerve, exposure is somehow compromised, as in the myocutaneous scalp flap technique, because of the bulky temporalis muscle raised together with the scalp flap. In addition, zygomatic arch exposure is extremely difficult.

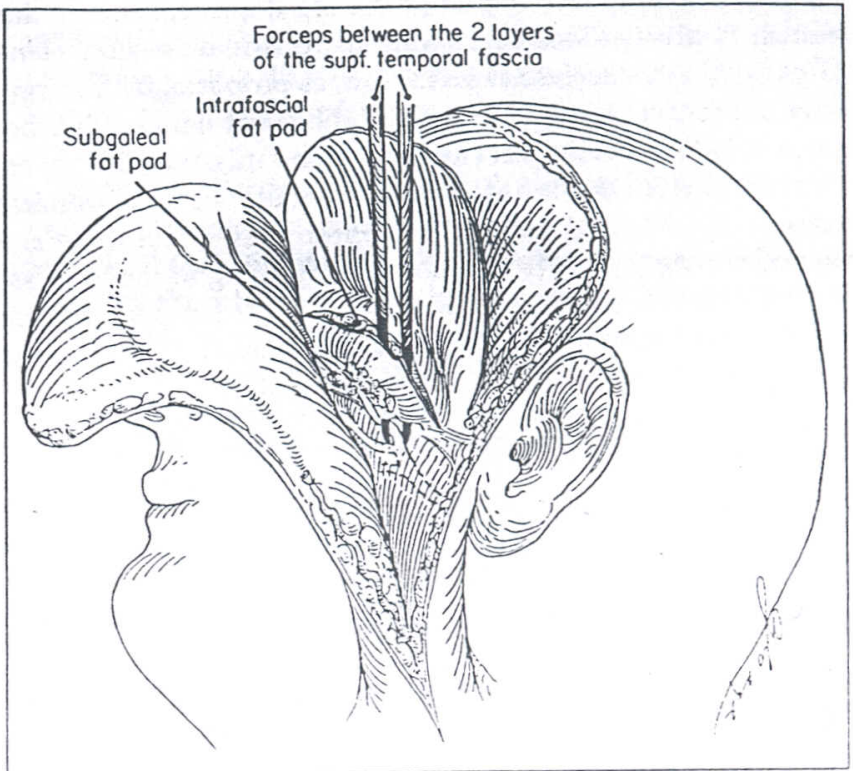

FIGURE 6. When dissection is performed in between the two layers of the superficial temporal fascia, it is easy, especially below the zygomatic arch, to injure the temporal branch of the facial nerve.

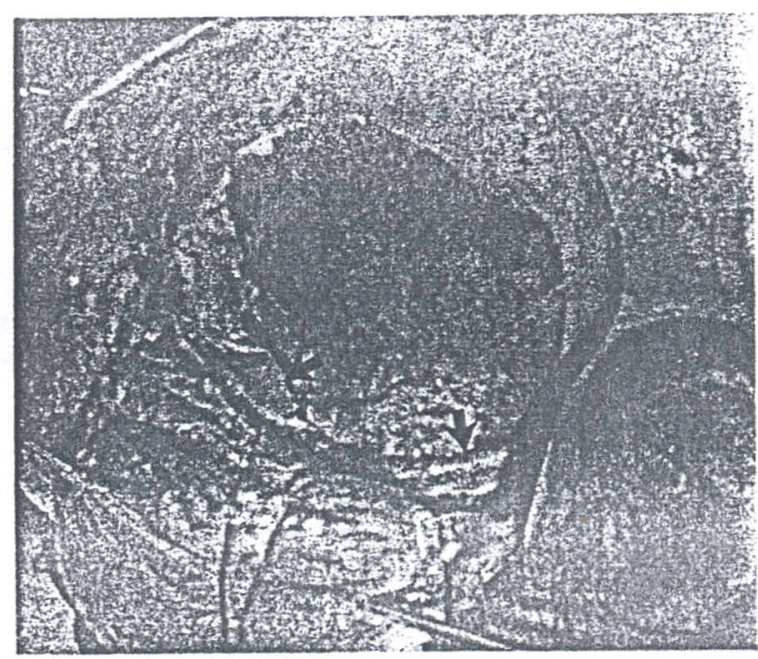

FIGURE 7. Anatomical specimen shows the proposed surgical dissection raising a combined scalp-superficial temporal fascia flap. Note the generous exposure of the zygomatic arch and of the frontozygomatic bone and the forceps holding the superficial temporal fascia. The deep fat pad is also evident.

\section{Practical implications of the present study}

Our study has shown that a frontotemporal scalp dissection that raises the superficial temporal fascia together with the scalp starting posterior or immediately anterior to the superficial temporal artery stem (Fig. 7) has virtually no chance of 
injuring the temporal branch of the facial nerve, because this branch is always located superficial to this dissection plane (Figs. 3-5). An interfascial dissection, as proposed by Yasargil, may injure the temporal branch of the facial nerve either because it is technically incorrect when one mistakes the superficial fat pad for the intrafascial one or because of anatomical reasons. Anatomical reasons come into play when there is a recurrent twig for the frontalis muscle running in the intrafascial fat pad or when the terminal twigs of the temporal branch of the facial nerve run in the subgaleal space posterior to the anterior one-fourth of the temporalis muscle.

\section{CONCLUSIONS}

The temporal branch of the facial nerve pierces the parotidomasseteric fascia below the zygomatic arch. This branch travels first in the subcutaneous tissue and then above the zygomatic arch, in the subgaleal space. The temporal branch divides into a frontal, a temporal, and a posterior ramus soon after it pierces the parotidomasseteric fascia. The course of the twigs of the temporal branch of the facial nerve in the subgaleal space displays a great degree of variability, with the location of the twigs being at times posterior to the anterior one-fourth of the temporalis muscle. Occasionally, a twig for the frontalis muscle may run in between the two layers of the superficial temporal fascia. Because of these findings (anteroposterior variability of the terminal twigs of the temporal branch of the facial nerve and recurrent intrafascial twig), Yasargil's interfascial dissection may at times fail. A combined frontotemporal scalp/ superficial temporal fascia dissection started posterior or immediately anterior to the superficial temporal artery is anatomically suited to preserve the temporal branch of the facial nerve.

Received, December 18, 1992.

Accepted, June 2, 1993.

Reprint requests: Mario Ammirati, M.D., Division of Neurosurgery, Room 74-140 CHS, University of California, Los Angeles, 10833 Le Conte Avenue, Los Angeles, CA 90024-6901.

\section{REFERENCES}

1. Al-Kayat A, Bramley P: A modified pre-auricular approach to the temporomandibular joint and malar arch. Br J Oral Surg 17:91103, 1979.

2. Al-Mefty O: Supraoibital-pterional approach to the skull base lesions. Neurosurgery 21:474-477, 1987.

3. Al-Mefty O, Anand VK: Zygomatic approach to skull-base lesions. J Neurosurg 73:668-673, 1990.

4. Ammirati M, Ma J, Becker D, Black K, Cheatham M, Bloch J: Transzygomatic approach to the tentorial incisura: Surgical anatomy. Skull Base Surg 2:161-166, 1992.

5. Aoki N: Incision of facial nerve branch at aneurysm surgery. J Neurosurg 66:482, 1987 (letter).

6. Baker DC, Conley J: Avoiding facial nerve injuries in rhytidectomy. Plast Reconstr Surg 64: 781-795, 1979.

7. Bernstein L, Nelson RH: Surgical anatomy of the extraparotid distribution of the facial nerve. Arch Otolaryngol 110:177-183, 1984.

8. Correia PC, Zani R: Surgical anatomy of the facial nerve, as related to ancillary operations in rhytidoplasty. Plast Reconstr Surg 52: 549-552, 1973.
9. Crowell RM, Ojemann RG: Surgical treatment of anterior comunicating artery aneurysms, in Schmidek $\mathrm{HH}$, Sweet WH (eds): Operative Neurosurgical Techniques, New York, Grune \& Stratton, 1982, vol 2, pp 829-854.

10. Fox JL: Cranial anatomy and the cranial flap, in Fox JL (ed): Atlas of Neurosurgical Anatomy. The Pterional Perspective. New York, Springer-Verlag, 1989, pp 37-41.

11. Fujitsu K, Kuwabara T: Zygomatic approach for lesions in the interpeduncular cistern. J Neurosurg 62:340-343, 1985.

12. Furnas DW: Landmark for the trunk and the temporo-facial division of the facial nerve. Br J Surg 52:694-696, 1965.

13. Gates GA: The lateral facial approach to the nasopharynx and infratemporal fossa. Otolaryngol Head Neck Surg 99:321-325, 1988.

14. Gray's Anatomy. Williams PC, Warwick R (eds). Philadelphia, W.B. Saunders Co., 1980, ed 36, pp 530, 534.

15. Ishikawa Y: An anatomical study on the distribution of the temporal branch of the facial nerve. J Craniomaxillofac Surg 18:287292, 1990.

16. Jane JA, Park TS, Pobereskin LH, Winn HR, Butler AB: The supraorbital approach. Technical note. Neurosurgery 11:537-542, 1987.

17. Kreutziger KL: Surgery of the temporomandibular joint. I. Surgical anatomy and surgical incisions. Oral Surg Oral Med Oral Path 58:637-646, 1984

18. Labbé D, Hubert P, Rigot-Jolivet M, Madjidi A: Subperiosteal subtemporal approach: Technique and applications. Neurosurgery 30:744-747, 1992.

19. Lang J: Clinical Anatomy of the Head. Neurocranium-Orbit-Craniocervical Regions. Berlin, Springer-Verlag, 1983, p 104.

20. Liebman EP, Webster RC, Berger AS, Vecchia MD: The frontalis nerve in the temporal brow lift. Arch Otolaryngol 108:232-235, 1982.

21. Ljungreen B, Fox JL: History of the pterional approach, in Fox JL (ed): Atlas of Neurosurgical Anatomy. The Ptirional Perspective. New York, Springer-Verlag, 1989, pp 1-9.

22. Pitanguy I, Ramos AS: The frontal branch of the facial nerve: The importance of its variations in face lifting. Plast Reconstr Surg 38:352-356, 1966.

23. Robbins TH: The protection of the frontal branch of the facial nerve in face-lift surgery. Br J Plast Surg 34:95-96, 1981.

24. Schmidek HH: Surgical management of aneurysms of the internal carotid: Posterior communicating, anterior choroidal and bifurcation aneurysms, in Schmidek HH, Sweet WH (eds): Operative Neurosurgical Techniques. New York, Grune \& Stratton, 1982, vol 2, pp 855-867.

25. Stuzin JM, Wagstrom L, Kawamoto HK, Wolfe SA: Anatomy of the frontal branch of the facial nerve: The significance of the temporal fat pad. Plast Reconstr Surg 83:265-271, 1989.

26. Testut L, Jacob O: Trattato di Anatomia Topografica. Con applicazioni medico chirurgiche. Torino, UTET, 1967, vol 1, p 53.

27. Uttley D, Archer DJ, Marsh HT, Bell BA: Improved access to lesions of the central skull base by mobilization of the zygoma: Experience with 54 cases. Neurosurgery 28:99-104, 1991.

28. Yasargil MG: Interfascial pterional (frontotemporosphenoidal) craniotomy, in Yasargil MG (ed): Microneurosurgery. New York, Georg Thieme Verlag, 1984, vol 1, pp 217-220.

29. Yasargil MG, Reichman MV, Kubik S: Preservation of frontotemporal branch of the facial nerve using the interfascial temporalis flap for pterional craniotomy. Technical article. J Neurosurg 67: 463-466, 1987. 


\section{COMMENT}

removes bone inferiorly from the most anterior burr hole. In-

Ammirati and his colleagues have demonstrated the surgical anatomy of the temporal branch of the facial nerve above the zygomatic arch. Neurosurgeons work on this area on a regular basis, and it is beneficial to see more detailed attention paid to the extracranial anatomy here.

The next step will be to apply the principle outlined by the authors in the operating room during a typical frontotemporal, or pterional, craniotomy. There still may be significant problems regarding injury to the temporal branch of the facial nerve even after peeling away the superficial layer of the temporalis muscle fascia. The branches of the nerve may be injured as one jury may occur with electrocoagulation of nearby vessels. The nerve may be stretched by traction from sutures or fishhook retractors.

In my own experience, when the frontalis branch of this nerve is injured, it usually returns to function in about 6 months. More significant cosmetic defects occur when the branch to the orbicularis oculi and corrugator supercilii muscles occurs. This is certainly less common than injury to the frontalis branch; but when it does occur, there is a significant lid lag. I look forward to seeing the practical results of this study.

John L. Fox Omaha, Nebraska 